\title{
QUANTUM CHANNELS IN NONLINEAR OPTICAL PROCESSES
}

\author{
DAVID S. BRADSHAW AND DAVID L. ANDREWS* \\ Nanostructures and Photomolecular Systems, School of Chemical Sciences, \\ University of East Anglia, Norwich NR4 7TJ, United Kingdom.
}

Quantum electrodynamics furnishes a new type of representation for the characterisation of nonlinear optical processes. The treatment elicits the detailed role and interplay of specific quantum channels, information that is not afforded by other methods. Following an illustrative application to the case of Rayleigh scattering, the method is applied to second and third harmonic generation. Derivations are given of parameters that quantify the various quantum channels and their interferences; the results are illustrated graphically. With given examples, it is shown in some systems that optical nonlinearity owes its origin to an isolated channel, or a small group of channels.

Keywords: Nonlinear optics; harmonic generation; quantum electrodynamics.

\section{Introduction}

Since the first utilisation of nonlinear materials for laser frequency conversion - and the discovery that many could be unexpectedly robust under the high intensities of laser light required - the field has developed and branched into strikingly diverse technological avenues. New materials with increasingly optimised properties are often reported, particularly in connection with photoactive devices, optical sensors, telecommunications systems and optical data storage amongst others. To inform the devising of new, specially tailored nonlinear optical materials for these and other applications, accurate methods are required for calculating the salient operating characteristics. ${ }^{1-7}$ With the development of a graph theoretic approach for such computations, ${ }^{8}$ it has become much more straightforward to identify and quantify the significance of discrete quantum channels, contributing to specific nonlinear optical effects. The term quantum channel signifies a quantum mechanically distinct sequence of radiation-matter coupled states, connecting the initial and final optical states, each generating directly additive contributions to the material response tensor (e.g. hyperpolarisability). The 
method is based on the construction of a graph which, for any photon-based process, affords it a unique and complete representation. Any such graph can be interpreted as a state-sequence network connecting the initial and final system states. As calculational aids, these graphs have several key advantages over the more familiar (Feynman) time-ordered diagrams commonly employed: (i) the connectivity of different quantum channels is readily identifiable; (ii) salient parameters for states that feature in several channels need only be computed once; (iii) all time-orderings for a particular process are embedded in a single graph. Based on these state-sequence diagrams, a very recent report ${ }^{9}$ offers a significantly clearer perspective (compared to earlier semiclassical work) on the photophysics underlying nonlinear optical response.

In this paper we develop and illustrate application of the new methodology to the detailed characterisation of materials for laser frequency conversion. ${ }^{10,11}$ We also introduce a new illustrative tool to exhibit the relevance and degree of interference between the various quantum channels that contribute to frequency conversion. These are features that have, until now, rarely been reported - but which can play a decisive role in determining optical characteristics. Choosing a suitable model to illustrate the technique we show, using graphical representations, how quantum channels interfere both constructively and destructively; we demonstrate not only how such interferences can be identified, but also quantified according to process and frequency range. Amongst other advantages, the immediacy and discriminatory power of these representations commends their possible use as guides to the accuracy of calculational approximations.

\section{Theoretical basis}

The correct description of any nonlinear optical medium under the influence of electromagnetic radiation properly requires the employment of quantum field theory. Nonetheless there are two distinct approaches; often the matter is treated quantum mechanically and the radiation as a classical field (semiclassical theory, SCT); this has success in many applications but notably fails in others such as spontaneous emission (normal fluorescence, phosphorescence etc.). Alternatively, quantum electrodynamics (QED) can be used. QED describes both the matter and the radiation quantum mechanically; it has excellent agreement with experiment and is the only theory to fundamentally 
support the concept of a photon. ${ }^{12}$ Since the quantum channels that mediate the production of optical harmonics are specifically determined by different sequences of photon creation and annihilation in the nonlinear medium, it is QED that is the theory of choice for the following development.

For the systems discussed in this paper, it is sufficiently accurate to develop theory in terms of non-relativistic QED. As a result, since intra-atomic Coulomb binding energies are much greater than the coupling to radiation, matter-photon interactions are treated by perturbation methods. ${ }^{13}$ The following Hamiltonian for non-relativistic QED is directly amenable to multipolar development:

$$
H=H_{\mathrm{rad}}+H_{\mathrm{mat}}+H_{\mathrm{int}}
$$

Eq. (1) comprises: (i) the radiation Hamiltonian, $H_{\text {rad }}$; (ii) the matter Hamiltonian, $H_{\text {mat }}$ and; (iii) the interaction Hamiltonian, $H_{\mathrm{int}}$. The quantum representation of the radiation field engenders the concept of vacuum fluctuations, the source of the zero-point energy associated with a vacuum. Detailed calculations show that such fluctuations are responsible for Casimir interactions and contributes to the Lamb shift, for example. ${ }^{14}$ Equally, with the promotion of the radiation field to operator status in QED, $H_{\text {int }} \neq 0$ even when no radiation is present; as such the theory successfully accounts for spontaneous emission, a result which SCT notably fails to deliver. The rate, $\Gamma$, of any optical or other electromagnetic interaction is found from Fermi's Golden Rule ${ }^{15}$;

$$
\Gamma=\frac{2 \pi}{\hbar}\left|M_{F I}\right|^{2} \delta\left(E_{I}-E_{F}\right)
$$

where $\delta\left(E_{I}-E_{F}\right)$ is the Dirac delta function - which equals zero unless the initial energy, $E_{I}$, equals the final energy, $E_{F}$, and $M_{F I}$ is the quantum amplitude coupling the initial and final states. The latter is determined by time-dependent perturbation theory, generally cast as the following infinite series; 


$$
\begin{aligned}
M_{F I} & =\left\langle F\left|H_{\mathrm{int}}\right| I\right\rangle+\sum_{R} \frac{\left\langle F\left|H_{\mathrm{int}}\right| R\right\rangle\left\langle R\left|H_{\mathrm{int}}\right| I\right\rangle}{\left(E_{I}-E_{R}\right)}+\sum_{R, S} \frac{\left\langle F\left|H_{\mathrm{int}}\right| S\right\rangle\left\langle S\left|H_{\mathrm{int}}\right| R\right\rangle\left\langle R\left|H_{\mathrm{int}}\right| I\right\rangle}{\left(E_{I}-E_{R}\right)\left(E_{I}-E_{S}\right)} \\
& +\sum_{R, S, T} \frac{\left\langle F\left|H_{\mathrm{int}}\right| T\right\rangle\left\langle T\left|H_{\mathrm{int}}\right| S\right\rangle\left\langle S\left|H_{\mathrm{int}}\right| R\right\rangle\left\langle R\left|H_{\mathrm{int}}\right| I\right\rangle}{\left(E_{I}-E_{R}\right)\left(E_{I}-E_{S}\right)\left(E_{I}-E_{T}\right)}+\ldots,
\end{aligned}
$$

where $|I\rangle$ and $|F\rangle$ represent the initial and final system states, respectively; the virtual system states are denoted by $|R\rangle,|S\rangle,|T\rangle \ldots$ and $E_{I}$ is the energy of the initial state. Moreover, successive terms relate to processes of progressively higher photonic order; for example, a description of the threephoton event of second harmonic generation (SHG) and four-photon event of third harmonic generation (THG) are determined from the third and fourth terms on the right-hand side of Eq. (3), respectively. Processes cannot involve both three and four photons (for example) and, thus, no crossterms within Eq. (2) will occur in the QED case. In contrast, the semiclassical description proves problematic at this juncture since apparently non-zero cross-terms remain which, in practice, have no physical significance. Returning to Eq. (3), each operation of $H_{\text {int }}$ on the state to its right effects transition to the state on its left; physically this signifies the annihilation or creation of a photon by the nonlinear medium. The possible sequences in which such fundamental processes occur, as the radiation-matter system moves from its initial state to the final state, represent the various quantum channels of the given process.

For the expository purpose of this paper, simplified two-level systems are chosen for the nonlinear media. Calculations thus invoke only data on the ground state, the single optically significant excited state and their coupling, not all states of the medium. It should nonetheless be emphasised that adoption of the two-level approximation is a quite separate matter from the calculational procedures we describe; our methods are completely amenable to systems with any number of optically significant states. As an illustrative example of the method the simplest elastic optical process, Rayleigh scattering (RS), will be examined first. This is followed by more detailed analysis of the successively more complex processes of SHG and THG. The paper concludes with a discussion section. 


\section{Rayleigh scattering}

In QED terms, Rayleigh or elastic light scattering is a process involving two matter-photon interactions; the annihilation of an incoming photon and the creation of another. Due to the timeenergy uncertainty principle, theory requires that this phenomenologically instantaneous event is cast in terms of the two co-present quantum channels of the state-sequence diagram of Fig. 1. In one channel, the annihilation of a photon effects a transition to a virtual excited matter state, which relaxes back to the ground state with the creation (emission) of a further photon. The emergent light has the same wave-vector magnitude (and energy) as the incoming photon. The second quantum channel differs in the sequential creation of a photon and annihilation. An expression for the polarisability tensor $\alpha_{i j}-$ which is proportional to the quantum amplitude of Rayleigh scattering - is derived from the second term of Eq. (3) and given by ${ }^{13,16}$ :

$$
\alpha_{i j}=\sum_{r}\left(\frac{\mu_{i}^{0 r} \mu_{j}^{r 0}}{E_{r 0}-\hbar \omega}+\frac{\mu_{j}^{0 r} \mu_{i}^{r 0}}{E_{r 0}+\hbar \omega}\right)
$$

Here, the implied summation convention for repeated Cartesian tensor indices is used, $\boldsymbol{\mu}^{0 r} \equiv\langle 0|\boldsymbol{\mu}| r\rangle$ denotes a transition dipole moment, $\hbar \omega$ is the energy of an input photon and $E_{r 0} \equiv E_{r}-E_{0}$, in which $r$ represents the virtual matter state. Furthermore, within Eq. (4), the first term corresponds to the upper quantum channel of Fig. 1 and the second term to the lower quantum channel. Mathematically these two channels signify the following routes between quantum states;

$$
\begin{gathered}
\text { Channel 1 } \Rightarrow\left|r_{0}^{1}\right\rangle \rightarrow\left|r_{1}^{1}\right\rangle \rightarrow\left|r_{2}^{1}\right\rangle, \\
\text { Channel 2 } \Rightarrow\left|r_{0}^{1}\right\rangle \rightarrow\left|r_{1}^{2}\right\rangle \rightarrow\left|r_{2}^{1}\right\rangle,
\end{gathered}
$$

where $\left|r_{k}^{l}\right\rangle$ denotes a system state displayed on the relevant state-sequence diagram: $k$ is the step number ( 0 being the initial state, represented on the left-hand side of the state-sequence diagram) and $l$ the vertex number (in numerical order from top to base). Eq. (4) involves a summation over all virtual states of the medium and hence allows the expression to be rewritten in the form ${ }^{17}$ : 


$$
\alpha_{i j}=\alpha_{i j}^{\mathrm{TLA}}+\alpha_{i j}^{\mathrm{BG}}
$$

Here, TLA denotes the two-level approximation and BG other background contributions. For any approximately two-level system, the second term on the right-hand side of Eq. (5) is insignificant and need not be considered further - this is an approximation that is unnecessary in many systems but which simplifies the present exposition. Therefore, setting $|r\rangle \subset\{|u\rangle,|0\rangle\}$ and $E_{r 0}=E_{u 0}$, Eq. (4) simplifies to:

$$
\alpha_{i j}^{\mathrm{TLA}}=\frac{\mu_{i}^{0 u} \mu_{j}^{0 u}}{E_{u 0}-\hbar \omega}+\frac{\mu_{i}^{0 u} \mu_{j}^{0 u}}{E_{u 0}+\hbar \omega}={ }^{(1)} \alpha_{i j}+{ }^{(2)} \alpha_{i j} .
$$

Note here that the terms involving the static dipole moment, $\boldsymbol{\mu}^{00}$, cancel out exactly. Moreover, the wavefunctions of the matter states $|0\rangle$ and $|u\rangle$ are both assumed as either non-degenerate or a suitable linear combination of degenerate states, and hence are characterized by real functions; the expression $\mu^{0 u} \equiv \mu^{u 0}$ then follows.

The rate equation (2) can be rewritten for the present application by splitting the quantum amplitude into two terms - the first term, ${ }^{(1)} M_{F I}$, corresponding to channel 1 and the second, ${ }^{(2)} M_{F I}$, to channel 2:

$$
\Gamma_{\mathrm{TOT}}^{\mathrm{RS}}=\frac{2 \pi}{\hbar}\left({ }^{(1)} M_{F I}+{ }^{(2)} M_{F I}\right)^{2} \delta\left(E_{f}-E_{i}\right)
$$

In terms of polarisabilities, accounting for ${ }^{(1)} M_{F I} \sim{ }^{(1)} \alpha_{i j}$ and ${ }^{(2)} M_{F I} \sim{ }^{(2)} \alpha_{i j}$, this expression is rewritten in the following simplified form:

$$
\Gamma_{\mathrm{TOT}}^{\mathrm{RS}} \sim\left({ }^{(1)} \alpha_{i j}+{ }^{(2)} \alpha_{i j}\right)^{2} .
$$


We now identify a matrix of contribution ratios, $\gamma_{n m}$, whose diagonal components represent the significance of single channels through the state-sequence diagram, and whose off-diagonal terms represent the quantum interference of differing channels - see Figs 2(a)-(c). Normalising the relative values by reference to the total rate (of elastic scattering, in this instance), only the individual polarizability contributions are of importance as other factors cancel in the ratios. Thus we secure the following expression, written in terms of ${ }^{(1)} \alpha$ and ${ }^{(2)} \alpha$, from which the three contribution ratios of Rayleigh scattering are generated;

$$
\gamma_{n m}^{\mathrm{RS}}=\frac{{ }^{(n)} M_{f i}{ }^{(m)} M_{f i}}{\left({ }^{(1)} M_{f i}+{ }^{(2)} M_{f i}\right)^{2}}=\frac{{ }^{(n)} \alpha_{i j}{ }^{(m)} \alpha_{k l}}{\left({ }^{(1)} \alpha_{i j}+{ }^{(2)} \alpha_{i j}\right)^{2}}=\frac{{ }^{(n)} \alpha^{(m)} \alpha}{\left({ }^{(1)} \alpha+{ }^{(2)} \alpha\right)^{2}},
$$

where $n, m=1$ or $2, \gamma_{21}^{\mathrm{RS}} \equiv \gamma_{12}^{\mathrm{RS}}$ and it is possible for the off-diagonal $\gamma_{n m}$ to have either positive or a negative values, respectively signifying constructive or destructive quantum interference. The validity of an apparent cancellation of Cartesian components in the last equality, in Eq. (9), is proven in Appendix A, where more detail is given on the derivation of the contribution ratio. Generated from Eq. (9), the contribution ratios are given as follows:

$$
\begin{gathered}
\gamma_{11}^{\mathrm{RS}}=\left(\frac{E_{u 0}+\hbar \omega}{2 E_{u 0}}\right)^{2}, \\
\gamma_{22}^{\mathrm{RS}}=\left(\frac{E_{u 0}-\hbar \omega}{2 E_{u 0}}\right)^{2}, \\
\gamma_{12}^{\mathrm{RS}}=\frac{\left(E_{u 0}+\hbar \omega\right)\left(E_{u 0}-\hbar \omega\right)}{\left(2 E_{u 0}\right)^{2}} .
\end{gathered}
$$

The results of Eq. (9) are readily quantified for input photons of various frequencies in the energy range spanning the optical range between $2 \times 10^{-18} \mathrm{~J}$ and $1 \times 10^{-20} \mathrm{~J}$ (wavelength range $99.3 \mathrm{~nm}$ to $19.8 \mu \mathrm{m})$, i.e. energies comparable to the given typical value of $E_{u 0}=8.15 \times 10^{-19} \mathrm{~J}(243.7 \mathrm{~nm})$. For present purposes photon energies are chosen not to match or very closely approach $E_{u 0}$, thus precluding complex resonance absorption considerations. To interpret the results, once the relevant 
values are inserted into Eqs (10)-(12), a graphical representation is constructed (Fig. 3). The main feature of the graph is that each line depicts the contribution due to the channel (or channels) illustrated by Figs 2 (a)-(c), i.e. lines [1,1], [2,2] and [1,2] relate to Eq. (10), (11) and (12) respectively. From the analysis of Fig. 3, it is observed that contributor [1,1] always gives the greatest contribution to the total scattering, except for incoming photon wavelengths approaching $20 \mu \mathrm{m}$; then all contributors are equivalent. Note that the line [1,2] includes the result for $\gamma_{12}^{\mathrm{RS}}$ and $\gamma_{21}^{\mathrm{RS}}$; accordingly contributor [1,2] features a contribution twice the value of the two 'diagonal' contributions at high photonic wavelengths. It is also observed that $[1,2]$ is the only term that may have a negative value - but this is apparent only at photon energies greater than $E_{u 0}$. The negative value signifies that there is destructive quantum interference between the two channels, i.e. a contribution that diminishes the efficiency of scattering.

\section{Second harmonic generation}

Second harmonic generation fundamentally involves three matter-photon interactions, in each of which two identical photons are annihilated and one frequency-doubled photon is created in the nonlinear medium. For this process the total energy of the two incoming photons, $2 \hbar \omega$, is equivalent to the energy of the outgoing photon, $\hbar \omega^{\prime}$; the mechanism is thus elastic. A single state-sequence diagram accommodates all possible pathways by which this process can proceed; this is illustrated by Fig. 4, and the three possible quantum channels are defined in Table 1. To determine the second-order hyperpolarisability of second harmonic generation, $\beta_{i j k}$, the third term of Eq. (3) is required. The derivation that then follows is considerably simplified by the use of a very recently proposed procedure. ${ }^{9}$ The result is reported as the familiar summation;

$$
\begin{aligned}
\beta_{i j k}=\sum_{r, s}( & \frac{\mu_{i}^{0 s} \mu_{j}^{s r} \mu_{k}^{r 0}}{\left(E_{s 0}-2 \hbar \omega\right)\left(E_{r 0}-\hbar \omega\right)}+\frac{\mu_{j}^{0 s} \mu_{i}^{s r} \mu_{k}^{r 0}}{\left(E_{s 0}+\hbar \omega\right)\left(E_{r 0}-\hbar \omega\right)} \\
& \left.+\frac{\mu_{j}^{0 s} \mu_{k}^{s r} \mu_{i}^{r 0}}{\left(E_{s 0}+2 \hbar \omega\right)\left(E_{r 0}+\hbar \omega\right)}\right),
\end{aligned}
$$


where $s$ denotes a second virtual matter state. Once again applying the two-level approximation, namely $|r\rangle,|s\rangle \subset\{|u\rangle,|0\rangle\}$, Eq. (13) becomes ${ }^{17-20}$ :

$$
\begin{aligned}
\beta_{i j k}^{\mathrm{TLA}} & =\frac{\mu_{i}^{0 u} \breve{d}_{j} \mu_{k}^{0 u}}{\left(E_{u 0}-2 \hbar \omega\right)\left(E_{u 0}-\hbar \omega\right)}+\frac{\mu_{j}^{0 u} \breve{d}_{i} \mu_{k}^{0 u}}{\left(E_{u 0}+\hbar \omega\right)\left(E_{u 0}-\hbar \omega\right)}+\frac{\mu_{j}^{0 u} \breve{d}_{k} \mu_{i}^{0 u}}{\left(E_{u 0}+2 \hbar \omega\right)\left(E_{u 0}+\hbar \omega\right)} \\
& ={ }^{(1)} \beta_{i j k}+{ }^{(2)} \beta_{i j k}+{ }^{(3)} \beta_{i j k} .
\end{aligned}
$$

Here, $\breve{\mathrm{d}}=\boldsymbol{\mu}^{u u}-\boldsymbol{\mu}^{00}$ is the static dipole displacement vector, i.e. the difference between the static dipole moments of the excited and ground matter states. The three terms ${ }^{(1)} \beta_{i j k},{ }^{(2)} \beta_{i j k}$ and ${ }^{\text {(3) }} \beta_{i j k}$ correspond to the channels 1, 2 and 3 (respectively); the latter are defined in Table 1. Again, we characterise contribution ratios as relating to an individual channel (quadratically determined from one hyperpolarisability term) or the quantum interference of differing channels (multiplying different terms). When the vectors $\breve{\mathbf{d}}$ and $\boldsymbol{\mu}^{0 u}$ are parallel, such that $\breve{\mathbf{d}}=\kappa \boldsymbol{\mu}^{0 u}$, then $\kappa \mu_{i}^{0 u} \mu_{j}^{0 u} \mu_{k}^{0 u} \equiv \mu_{i}^{0 u} \breve{d}_{j} \mu_{k}^{0 u} \equiv \mu_{j}^{0 u} \breve{d}_{i} \mu_{k}^{0 u} \equiv \mu_{j}^{0 u} \breve{d}_{k} \mu_{i}^{0 u}$ and the contribution ratio is independent of the Cartesian components $i j k$. Hence the following simple expression, written in terms of ${ }^{(1)} \beta{ }^{(2)} \beta$ and ${ }^{(3)} \beta$, that determines the six contribution ratios of SHG;

$$
\gamma_{n m}^{\mathrm{SHG}}=\frac{{ }^{(n)} \beta^{(m)} \beta}{\left(\sum_{i=1}^{3}{ }^{(i)} \beta\right)^{2}},
$$

where $n, m=1,2$ or 3 and $\gamma_{n m}^{\mathrm{SHG}} \equiv \gamma_{m n}^{\mathrm{SHG}}$. More intricate cases, in which $\breve{\mathbf{d}}$ and $\boldsymbol{\mu}^{0 u}$ are not parallel, are considered and discussed in the concluding Section.

For each of the expressions derived from Eq. (15), calculations are performed for photon input over the same wavelength range as previously for Rayleigh scattering, i.e. between $99.3 \mathrm{~nm}$ and 19.8 $\mu \mathrm{m}$. From an analysis of the corresponding graphical representation (Fig. 5) it is observed that at wavelengths greater than $20 \mu \mathrm{m}$ all contributions relating to a single channel contribute to an essentially equal amount, but the quantum interference of differing channels is approximately twice as 
significant; as before, this is due to the $\gamma_{n m}^{\mathrm{SHG}}$ being incorporated in the $\gamma_{m n}^{\mathrm{SHG}}$ result where $n \neq m$. In the region lower than $20 \mu \mathrm{m}$, the positive value of contributor [1,1] increasingly dominates as the wavelength decreases; [2,2] also increases but less rapidly, and [1,2] descends rapidly into negative results. Contributors [1,3], [2,3] and [3,3] are negligible and, hence, are not shown in Fig. 5. To summarise, it is found that quantum interference, i.e. [1,2], effectively erodes the strongly increasing $[1,1]$ contribution.

\section{Third harmonic generation}

Third harmonic generation entails the annihilation of three identical photons in a four matter-photon interaction process; again the process is elastic. The third-order hyperpolarisability of third harmonic generation, $\chi_{i j k l}$, arises from the fourth term of Eq. (3). To derive $\chi_{i j k l}$ the procedure of ref. 9 could again be employed but, given that the two-level approximation is again applied, it is expedient to use a calculational short-cut to the more complicated result. Proven in detail elsewhere, ${ }^{21}$ this method employs a reformulation algorithm that operates with permanent electric dipole moments as follows;

$$
\boldsymbol{\mu}^{u u} \rightarrow \boldsymbol{\mu}^{u u}-\boldsymbol{\mu}^{00}=\breve{\mathbf{d}} ; \quad \boldsymbol{\mu}^{00}=0 ;
$$

any transition dipole moment (here $\mu^{0 u}$ or $\mu^{u 0}$ ) is left unchanged. ${ }^{22}$ For THG, the sequence from the initial state to the final state proceeds via the three intermediate matter states, $|r\rangle,|s\rangle$ and $|t\rangle$, and is written $|0\rangle \rightarrow|r\rangle \rightarrow|s\rangle \rightarrow|t\rangle \rightarrow|0\rangle$. With the two-level approximation each of these intermediate states is either $|0\rangle$ or $|u\rangle$; thus the possible sequences are $00000,0 u 000,00 u 00,000 u 0,0 u u 00,0 u 0 u 0$, $00 u u 0,0 u u u 0$, where for example $0 u 0 u 0$ represents $\boldsymbol{\mu}^{0 u} \boldsymbol{\mu}^{u 0} \boldsymbol{\mu}^{0 u} \boldsymbol{\mu}^{u 0}$. Every sequence that includes the segment 00 is discarded, following Eq. (16); which leaves $0 u 0 u 0$ and $0 u u u 0$. Considering all channels through the state-sequence diagram of Fig. 6, the following eight-term expression is determined: 


$$
\begin{aligned}
\chi_{i j k l}^{\mathrm{TLA}}= & \frac{\mu_{i}^{0 u} \mu_{j}^{0 u} \mu_{k}^{0 u} \mu_{l}^{0 u}}{\left(E_{u 0}-3 \hbar \omega\right)(-2 \hbar \omega)\left(E_{u 0}-\hbar \omega\right)}+\frac{\mu_{i}^{0 u} \breve{d}_{j} \breve{d}_{k} \mu_{l}^{0 u}}{\left(E_{u 0}-3 \hbar \omega\right)\left(E_{u 0}-2 \hbar \omega\right)\left(E_{u 0}-\hbar \omega\right)} \\
& +\frac{\mu_{j}^{0 u} \mu_{i}^{0 u} \mu_{k}^{0 u} \mu_{l}^{0 u}}{\left(E_{u 0}+\hbar \omega\right)(-2 \hbar \omega)\left(E_{u 0}-\hbar \omega\right)}+\frac{\mu_{j}^{0 u} \breve{d}_{i} \breve{d}_{k} \mu_{l}^{0 u}}{\left(E_{u 0}+\hbar \omega\right)\left(E_{u 0}-2 \hbar \omega\right)\left(E_{u 0}-\hbar \omega\right)} \\
& +\frac{\mu_{j}^{0 u} \mu_{k}^{0 u} \mu_{i}^{0 u} \mu_{l}^{0 u}}{\left(E_{u 0}+\hbar \omega\right)(+2 \hbar \omega)\left(E_{u 0}-\hbar \omega\right)}+\frac{\mu_{j}^{0 u} \breve{d}_{k} \breve{d}_{i} \mu_{l}^{0 u}}{\left(E_{u 0}+\hbar \omega\right)\left(E_{u 0}+2 \hbar \omega\right)\left(E_{u 0}-\hbar \omega\right)} \\
& +\frac{\mu_{j}^{0 u} \mu_{k}^{0 u} \mu_{l}^{0 u} \mu_{i}^{0 u}}{\left(E_{u 0}+\hbar \omega\right)(+2 \hbar \omega)\left(E_{u 0}+3 \hbar \omega\right)}+\frac{\mu_{j}^{0 u} \breve{d}_{k} \breve{d}_{l} \mu_{i}^{0 u}}{\left(E_{u 0}+\hbar \omega\right)\left(E_{u 0}+2 \hbar \omega\right)\left(E_{u 0}+3 \hbar \omega\right)} \\
= & \sum_{i=1}^{8}{ }^{(i)} \chi_{i j k l} .
\end{aligned}
$$

Here, ${ }^{(1)} \chi_{i j k l}+{ }^{(2)} \chi_{i j k l}$ relate to channel 1 (defined in Table 2); the only differences between the terms being the differing energies of the intermediate states. Similarly ${ }^{(3)} \chi_{i j k l}+{ }^{(4)} \chi_{i j k l},{ }^{(5)} \chi_{i j k l}+{ }^{(6)} \chi_{i j k l}$ and ${ }^{(7)} \chi_{i j k l}+{ }^{(8)} \chi_{i j k l}$ correspond to channels 2,3 and 4 respectively.

Following the procedure established in previous sections, the ten contribution ratios of THG are formed by considering the contributions relating either to a single channel or the quantum interference of differing channels. These ratios are generated from the following expression;

$$
\gamma_{n m}^{\mathrm{THG}}=\frac{\left({ }^{(2 n-1)} \chi+{ }^{(2 n)} \chi\right)\left({ }^{(2 m-1)} \chi+{ }^{(2 m)} \chi\right)}{\left(\sum_{i=1}{ }^{(i)} \chi\right)^{2}}
$$

where $n, m=1,2,3$ or 4 , and $\Gamma_{n m}^{\mathrm{THG}}=\Gamma_{m n}^{\mathrm{THG}}$. Again taking $\breve{\mathbf{d}}$ and $\boldsymbol{\mu}^{0 u}$ as parallel, it follows that $\mu_{i}^{0 u} \mu_{j}^{0 u} \mu_{k}^{0 u} \mu_{l}^{0 u} \equiv \mu_{i}^{0 u} \breve{d}_{j} \breve{d}_{k} \mu_{l}^{0 u} \equiv \mu_{j}^{0 u} \mu_{i}^{0 u} \mu_{k}^{0 u} \mu_{l}^{0 u}$ etc. and the ensuing results are independent of the Cartesian components $i j k l$. In calculating results from Eq. (18), the previous typical values are again employed. From analysis of Fig. 7, constructed to interpret the THG results, it is observed at photon energies descending from $990 \mathrm{~nm}$, that contributors [1,1] and [1,2] are the dominant positive and negative contributions, respectively. However at wavelengths below $570 \mathrm{~nm},[1,3]$ and $[2,2]$ prevail over [1,1]. Therefore, [1,1] is not always the greatest rate contributor as in the SHG case. 


\section{Discussion}

Previously we have assumed, purely for simplicity of exposition, that the key dipole moments (i.e. the transition moment $\boldsymbol{\mu}^{0 u}$ and the static dipole displacement vector $\breve{\mathbf{d}}$ for one optically prominent excitation) are parallel. Hence, all the numerators of Eqs (14) and (17) are directionally equivalent and none of the quantum channels can be excluded from the analysis (although the channels of negligible significance have been omitted from the relevant graphs). Our methods are of course applicable to systems where the key dipole moments are not parallel - though the expressions that result require another level of exploration. If, for example, we consider systems in which $\mu^{0 u}$ and $\breve{\mathbf{d}}$ are orthogonal then it is possible to isolate a single channel or small group of channels. Through the application of such conditions, it can transpire that numerous quantum channels are precluded for certain hyperpolarisabilities. First analysing SHG; if for example $\breve{\mathbf{d}}$ and $\boldsymbol{\mu}^{0 u}$ are oriented in material-defined $z$ - and $x$-directions, respectively, then channel 2 alone exists for the corresponding tensor $\beta_{z x x}-$ whereas (taking account of the $j, k$ symmetry) $\beta_{x(z x)}$ will involve only channels 1 and 3 . The analogous results for THG are presented in Table 3. In general, for various angles between $\mu^{0 u}$ and $\breve{\mathbf{d}}$, a significant reduction of quantum channels is apparent.

This paper has highlighted and corrected a number of observed misconceptions. It is surprising to find that, in the context of describing nonlinear optical properties, a significant number of papers ${ }^{23-29}$ continue the early practice of reporting material response in terms of static (hyper)polarizability values, defined solely by a scalar magnitude. This misrepresents the tensor character and disregards the dispersion characteristics of the optical materials. Our analysis, with its in-built capacity to distinguish between quantum contributions on the basis of both directional attributes and dispersion behaviour, forcefully demonstrates that: (i) there is in general more than one significant route between the initial state and the final state in nonlinear optical processes; (ii) not only do hyperpolarisabilities have highly significant dispersion characteristics, but the different quantum channels vary dramatically with differing photon wavelengths; (iii) in materials with suitable symmetry properties the tensor character of the hyperpolarisabilities leads to the possibility of selecting certain quantum channels. 
The method we have described represents a novel and highly efficient tool for both the calculation and visualisation of nonlinear features that are not commonly known. Whereas this work has discussed nonlinear optical response in terms of individual scattering centres and photons, it is possible to adapt the equations to bridge the gap between microscopic (particle) and macroscopic (bulk) theories. This will entail proper representation of the propagation characteristics of the laser input and harmonic output, as modified by the bulk characteristics of the medium through which they propagate, and the distinctive features associated with collective response of an ensemble of particles or scattering centres. We are hopeful that the fundamental studies presented here will enhance the investigation of nonlinear optical materials, and potentially improve their design.

* david.andrews@physics.org

\section{Acknowledgments}

We gratefully acknowledge financial support from the Engineering and Physical Sciences Research Council, and Leverhulme Trust. We thank R. D. Jenkins and L. C. Dávila Romero for many helpful comments.

\section{Appendix A}

From Eq. (2), for an $m$-photon process, the rate equation may be rewritten as ${ }^{11}$;

$$
\Gamma=\frac{2 \pi}{\hbar}\left|f^{(m)} \alpha_{i_{1} \ldots i_{m}}^{(m)} s_{i_{1} \ldots i_{m}}^{(m)}\right|^{2} \delta\left(E_{i}-E_{f}\right)
$$

where $\alpha_{i_{1} . . i_{m}}^{(m)}$ is a generalised form of the (hyper)polarisability tensor, written in the main text as $\alpha_{i_{1} i_{2}}^{(2)} \equiv \alpha_{i j}, \quad \alpha_{i_{1} \ldots i_{3}}^{(3)} \equiv \beta_{i j k}$ and $\alpha_{i_{1} \ldots i_{4}}^{(4)} \equiv \chi_{i j k l} ;$ also, $s_{i_{1} \ldots i_{m}}^{(m)}$ is the corresponding product of the photon polarisation unit vectors and $f^{(m)}$ is given by: 


$$
f^{(m)}=(\mathrm{i})^{m}\left(\frac{\hbar c k}{2 \varepsilon_{0} V}\right)^{\frac{m}{2}} \prod_{j=1}^{m-1}\left[(n-j+1)^{\frac{1}{2}}\right] .
$$

For example, the rate for Rayleigh scattering $(m=2)$ is found as;

$$
\Gamma_{\mathrm{TOT}}^{\mathrm{RS}}=\frac{2 \pi}{\hbar}\left|f^{(2)} \alpha_{c} s_{c}^{(2)}\right|^{2} \delta\left(E_{i}-E_{f}\right),
$$

where $c$ represents the $m$-Cartesian components, i.e. $i, j$. The polarisability, $\alpha_{c}$, may be expressed in terms of quantum channels, $q$, namely:

$$
\alpha_{c} \equiv \sum_{q}^{(q)} \alpha_{c}
$$

Thus, Eq. (A3) is rewritten in the explicit form;

$$
\Gamma_{\mathrm{TOT}}^{\mathrm{RS}}=\frac{2 \pi}{\hbar}\left|f^{(2)} \sum_{c} \sum_{q}{ }^{(q)} \alpha_{c} s_{c}^{(2)}\right|^{2} \delta\left(E_{i}-E_{f}\right) .
$$

Due to $s_{c}^{(2)}$ being independent of $q$, Eq. (A5) is re-expressed as;

$$
\Gamma_{\mathrm{TOT}}^{\mathrm{RS}}=\frac{2 \pi}{\hbar}\left|f^{(2)} \sum_{c} s_{c}^{(2)} \sum_{q}^{(q)} \alpha_{c}\right|^{2} \delta\left(E_{i}-E_{f}\right) .
$$

Accounting for the summation over index-repeated Cartesian components and also the quantum channels, i.e. taking the summation over $c$ and $c^{\prime}$ along with $q$ and $q^{\prime}$, Eq. (A6) emerges as;

$$
\Gamma_{\mathrm{TOT}}^{\mathrm{RS}}=\frac{2 \pi}{\hbar}\left(f^{(2)} \bar{f}^{(2)} \sum_{c} \sum_{c^{\prime}} s_{c}^{(2)} \bar{s}_{c^{\prime}}^{(2)} \sum_{q} \sum_{q^{\prime}}{ }^{(q)} \alpha_{c}{ }^{\left(q^{\prime}\right)} \bar{\alpha}_{c^{\prime}}\right) \delta\left(E_{i}-E_{f}\right) .
$$


The contribution ratio, i.e. the rate contribution divided by the total rate, is independent of all but the polarisability part of equation (A7), and is generally written as;

$$
\gamma_{n m}^{\mathrm{RS}}=\frac{{ }^{(n)} \alpha^{(m)} \bar{\alpha}}{\sum_{q} \sum_{q^{\prime}}{ }^{(q)} \alpha^{\left(q^{\prime}\right)} \bar{\alpha}},
$$

where $n$ and $m=1$ or 2 (equivalently for $q$ and $q^{\prime}$ ); thus Eq. (A8) leads to Eq. (9). By examination of Eq. (6) it is apparent that the transition dipole moments are the only non-scalar factors (variables with Cartesian components). As all dipole moments cancel out in the contribution ratios, the scalar form of Eq. (A8) emerges. A scalar form for the contribution ratios of SHG and THG is also achieved if $\mathbf{d}$ and $\mu^{0 u}$ are parallel, as every hyperpolarisability numerator becomes identical. 


\section{References}

1. Z. Li, K. Wu, G. Su and Y. He, Opt. Mat. 20, 295 (2002).

2. L. Jensen, K. O. Sylvester-Hvid, K. V. Mikkelsen and P.-O. Åstrand, J. Phys. Chem. A 107, 2270 (2003).

3. K. Yanagi, T. Kobayashi and H. Hashimoto, Phys. Rev. B 67, 115122 (2003).

4. A. Ye, S. Patchkovskii and J. Autschbacha, J. Chem. Phys. 127, 074104 (2007).

5. M. Nakano, H. Nagai, H. Fukui, K. Yoneda, R. Kishi, H. Takahashi, A. Shimizu, T. Kubo, K. Kamada, K. Ohta, B. Champagne and E. Botek, Chem. Phys. Letts. 467, 120 (2008).

6. A. Mendoza-Garcia, J. L. Paz, M. Gorayeb, A. J. Hernandez, E. Castro and P. Martin, J. Nonl. Opt. Phys. Mat. 17, 511 (2008).

7. O. Loboda, R. Zaleśny, A. Avramopoulos, J.-M. Luis, B. Kirtman, N. Tagmatarchis, H. Reis and M. G. Papadopoulos, J. Phys. Chem. A 113, 1159 (2009).

8. R. D. Jenkins, D. L. Andrews and L. C. Dávila Romero, J. Phys. B: Atom. Molec. Opt. Phys. 35, 445 (2002).

9. D. L. Andrews and D. S. Bradshaw, Eur. J. Phys. 30, 239 (2009).

10. R. W. Boyd, Nonlinear Optics, 2nd edn. (Academic, New York, 2002).

11. D. L. Andrews and P. Allcock, Optical Harmonics in Molecular Systems (Wiley-VCH, Weinheim, 2002).

12. T. Kinoshita, Quantum Electrodynamics (World Scientific, Singapore, 1990).

13. D. P. Craig and T. Thirunamachandran, Molecular Quantum Electrodynamics (Dover, Mineola, New York, 1998).

14. P. W. Milonni, The Quantum Vacuum: An Introduction to Quantum Electrodynamics (Academic, San Diego, 1993).

15. E. Fermi, Nuclear Physics (University of Chicago Press, Chicago, 1950).

16. D. L. Andrews and K. P. Hopkins, Adv. Chem. Phys. 77, 39 (1990).

17. D. L. Andrews and W. J. Meath, J. Phys. B: At. Mol. Phys. 26, 4633 (1993).

18. J. L. Oudar and D. S. Chemla, J. Chem. Phys. 66, 2664 (1977).

19. J. L. Oudar, J. Chem. Phys. 67, 446 (1977).

20. R. Wortmann, P. Krämer, C. Glania, S. Lebus and N. Detzer, Chem. Phys. 173, 99 (1993). 
21. D. L. Andrews, L. C. Dávila Romero and W. J. Meath, J. Phys. B: At. Mol. Opt. Phys. 32, 1 (1999).

22. G. Juzeliūnas, D. L. Andrews and L. C. Dávila Romero, Phys. Rev. A 68, 043811 (2003).

23. S. Sharma, P. Gangopadhyay, A. Swathi and T. P. Radhakrishnan, Phys. Chem. Chem. Phys. 2, 1147 (2000).

24. Y. Zhang, W. Zhu, W. Wang, H. Tian, J. Su and W. Wang, J. Mater. Chem. 12, 1294 (2002).

25. H. Hou, Y. Wei, Y. Song, Y. Zhu, L. Li and Y. Fan, J. Mater. Chem. 12, 838 (2002).

26. J.-R. Gao, Q. Wang and L.-B. Cheng, Mat. Lett. 57, 761 (2002).

27. K. Ohta, S. Yamada, T. Tanaka and K. Kamada, Mol. Phys. 101, 315 (2003).

28. M. Nakano, H. Nagai, H. Fukui, K. Yoneda, R. Kishi, H. Takahashi, A. Shimizu, T. Kubo, K. Kamada, K. Ohta, B. Champagne, E. Botek, Chem. Phys. Lett. 467, 120 (2008).

29. B. Champagne and M. Spassova, Chem. Phys. Lett. 471, 111 (2009). 


\section{Figure legends}

Fig. 1: State-sequence diagram signifying Rayleigh scattering. The upper channel involves one photon (of energy $\hbar \omega$ ) annihilation then one photon creation (with $\hbar \omega^{\prime}$ ), the lower channel the converse order. Here, $\omega \equiv \omega^{\prime}$ since the process is elastic, an empty circle represents a ground matter state and the filled circles a virtual intermediate state (denoted by $r$ ). The step number, $k$, values are also presented on the figure.

Fig. 2: State-sequence diagrams representing the contributors: $(a)[1,1],(b)[2,2]$ and $(c)[1,2]$.

Fig. 3: Graphical representation of Rayleigh scattering. This shows a change in the magnitude of contributors $[1,1],[1,2]$ and $[2,2]$ with varying input photon frequency.

Fig. 4: $\quad$ State-sequence diagram signifying second harmonic generation. Here, as Fig. 1 except $2 \omega \equiv \omega^{\prime}$ and $s$ denotes a second virtual intermediate state.

Fig. 5: Graphical representation of second harmonic generation displaying contributors $[1,1]$, $[1,2]$ and $[2,2]$. The negligible contributors are omitted.

Fig. 6: State-sequence diagram signifying third harmonic generation. Here, as Fig. 4 except $3 \omega \equiv \omega^{\prime}$ and $t$ denotes a third virtual intermediate state.

Fig. 7: Graphical representation of third harmonic generation displaying contributors $[1,1]$, $[1,2],[1,3],[2,2],[2,3]$ and $[3,3]$. The negligible contributors are omitted.

Table 1: Three possible channels through the state-sequence diagram of SHG.

Table 2: $\quad$ Four possible channels through the state-sequence diagram of THG.

Table 3: For third harmonic generation, an example of channel isolation for a medium with an orthogonal transition dipole moment and dipole displacement vector. 


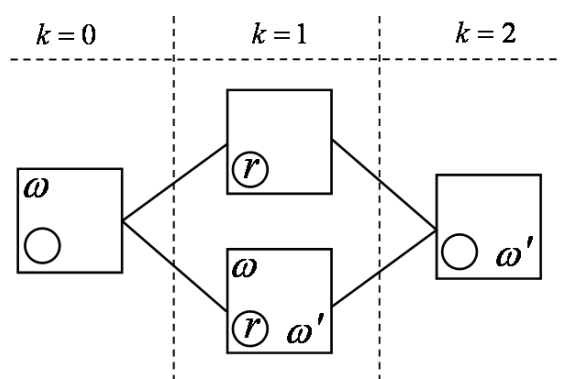

Fig. 1 


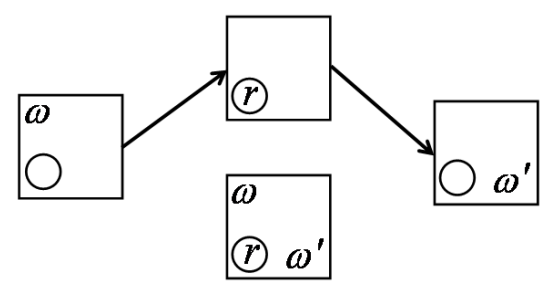

Fig. 2(a)

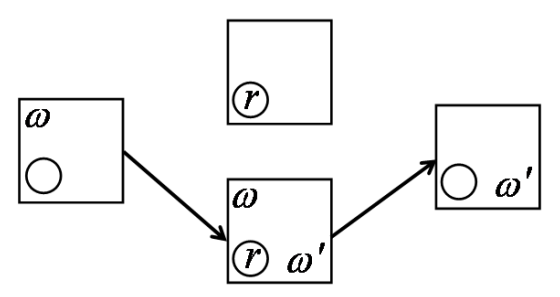

Fig. 2(b) 


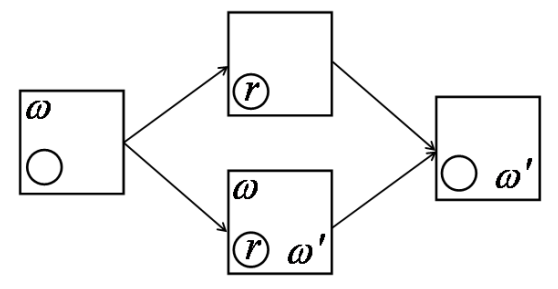

Fig. 2(c) 


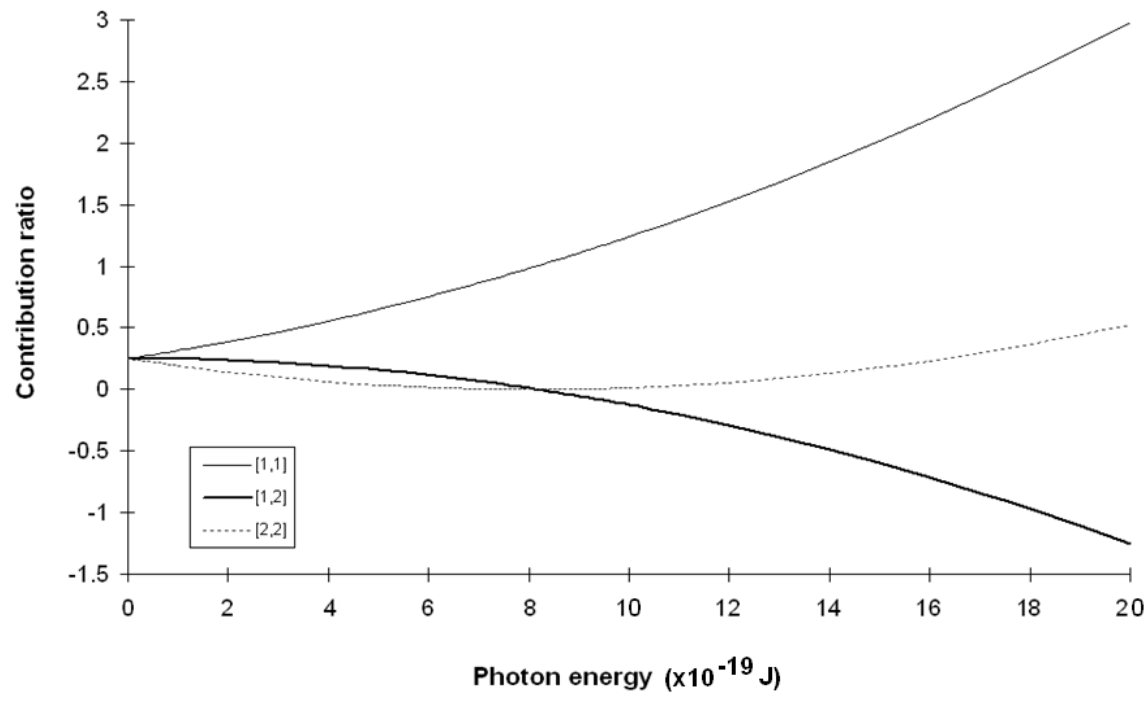

Fig. 3 


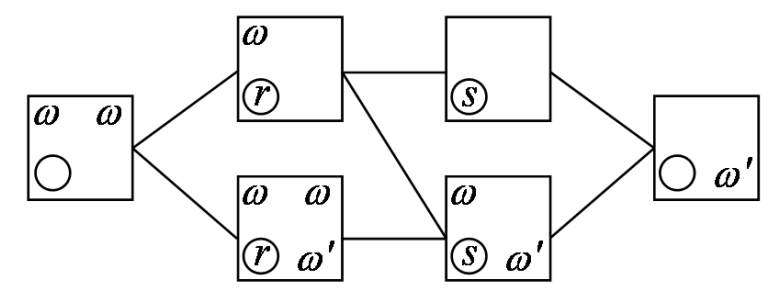

Fig. 4 


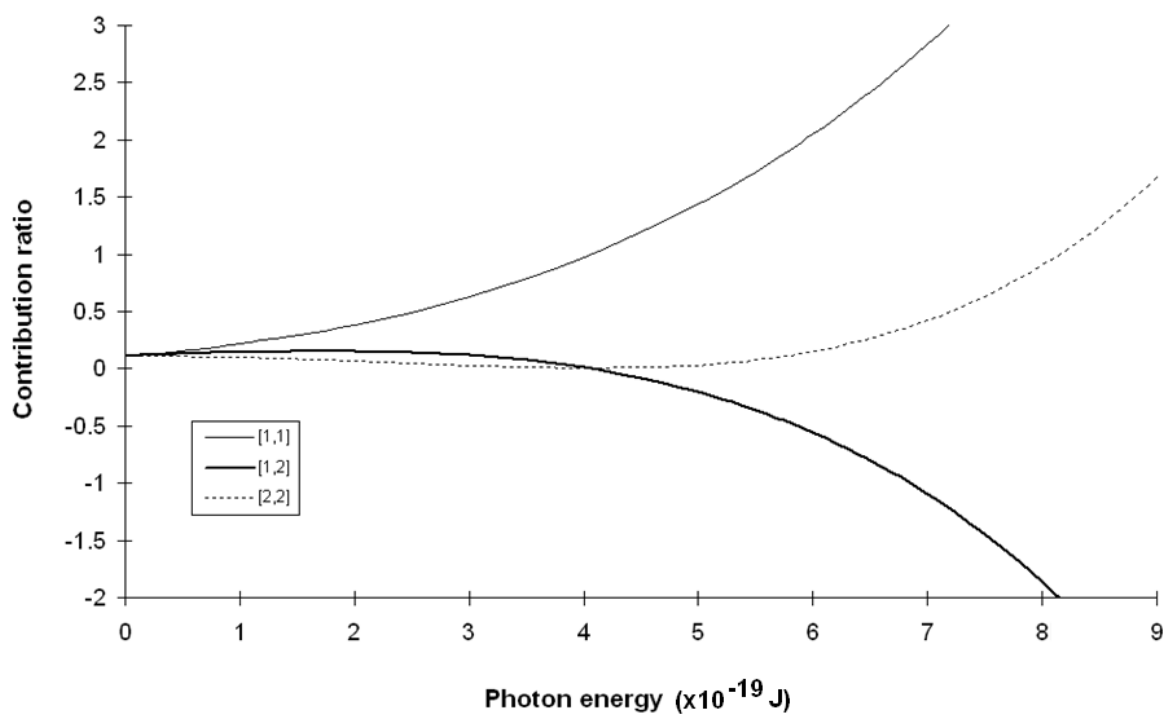

Fig. 5 


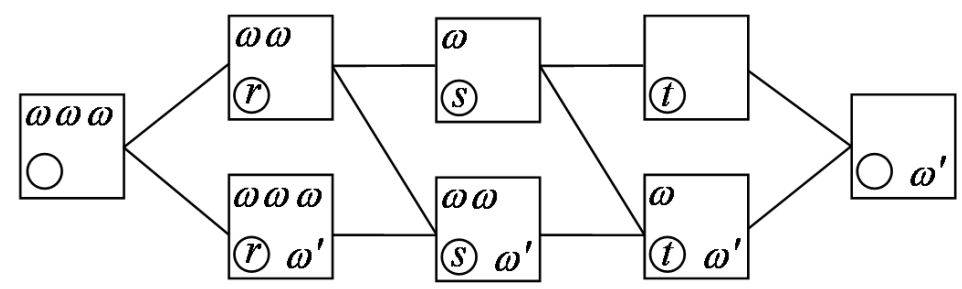

Fig.6 


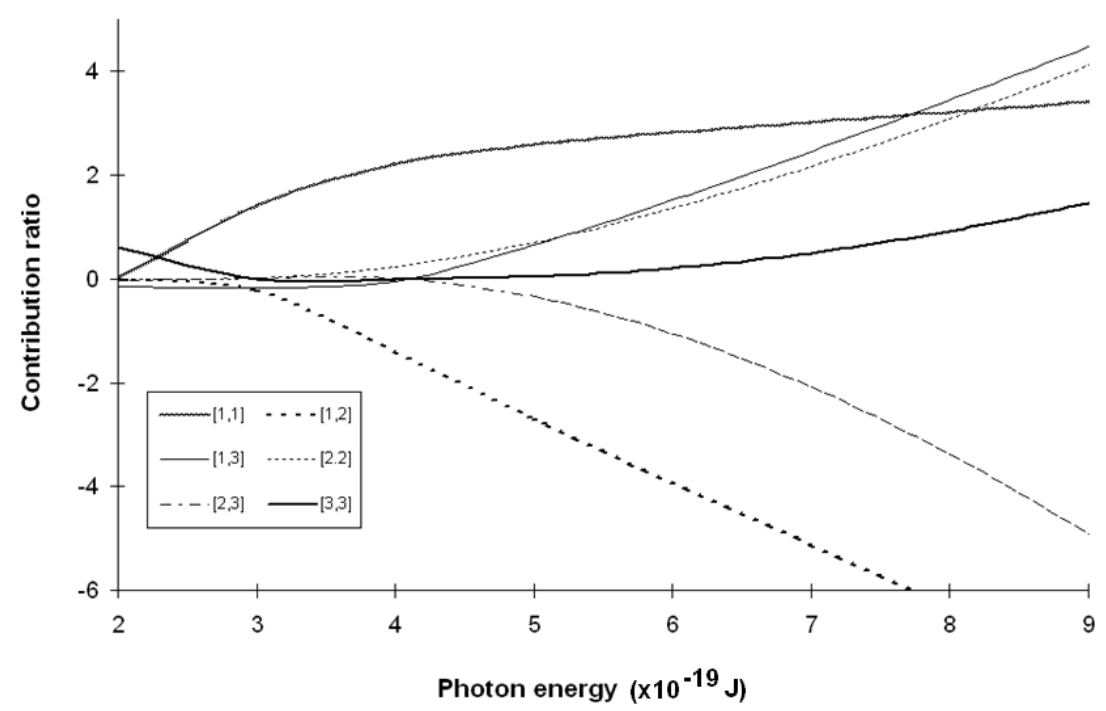

Fig. 7 


\begin{tabular}{|c|c|c|}
\hline Channel & Perturbation sequence & Mode occupancy \\
\hline 1 & $\left|r_{0}^{1}\right\rangle \rightarrow\left|r_{1}^{1}\right\rangle \rightarrow\left|r_{2}^{1}\right\rangle \rightarrow\left|r_{3}^{1}\right\rangle$ & $(\omega, \omega) \rightarrow(\omega) \rightarrow() \rightarrow\left(\omega^{\prime}\right)$ \\
\hline 2 & $\left|r_{0}^{1}\right\rangle \rightarrow\left|r_{1}^{1}\right\rangle \rightarrow\left|r_{2}^{2}\right\rangle \rightarrow\left|r_{3}^{1}\right\rangle$ & $(\omega, \omega) \rightarrow(\omega) \rightarrow\left(\omega, \omega^{\prime}\right) \rightarrow\left(\omega^{\prime}\right)$ \\
\hline 3 & $\left|r_{0}^{1}\right\rangle \rightarrow\left|r_{1}^{2}\right\rangle \rightarrow\left|r_{2}^{2}\right\rangle \rightarrow\left|r_{3}^{1}\right\rangle$ & $(\omega, \omega) \rightarrow\left(\omega, \omega, \omega^{\prime}\right) \rightarrow\left(\omega, \omega^{\prime}\right) \rightarrow\left(\omega^{\prime}\right)$ \\
\hline
\end{tabular}

Table 1. 


\begin{tabular}{|c|c|c|}
\hline Channel & Perturbation sequence & Mode occupancy \\
\hline 1 & $\left|r_{0}^{1}\right\rangle \rightarrow\left|r_{1}^{1}\right\rangle \rightarrow\left|r_{2}^{1}\right\rangle \rightarrow\left|r_{3}^{1}\right\rangle \rightarrow\left|r_{4}^{1}\right\rangle$ & $(\omega, \omega, \omega) \rightarrow(\omega, \omega) \rightarrow(\omega) \rightarrow() \rightarrow\left(\omega^{\prime}\right)$ \\
\hline 2 & $\left|r_{0}^{1}\right\rangle \rightarrow\left|r_{1}^{1}\right\rangle \rightarrow\left|r_{2}^{1}\right\rangle \rightarrow\left|r_{3}^{2}\right\rangle \rightarrow\left|r_{4}^{1}\right\rangle$ & $(\omega, \omega, \omega) \rightarrow(\omega, \omega) \rightarrow(\omega) \rightarrow\left(\omega, \omega^{\prime}\right) \rightarrow\left(\omega^{\prime}\right)$ \\
\hline 3 & $\left|r_{0}^{1}\right\rangle \rightarrow\left|r_{1}^{1}\right\rangle \rightarrow\left|r_{2}^{2}\right\rangle \rightarrow\left|r_{3}^{2}\right\rangle \rightarrow\left|r_{4}^{1}\right\rangle$ & $(\omega, \omega, \omega) \rightarrow(\omega, \omega) \rightarrow\left(\omega, \omega, \omega^{\prime}\right) \rightarrow\left(\omega, \omega^{\prime}\right) \rightarrow\left(\omega^{\prime}\right)$ \\
\hline 4 & $\left|r_{0}^{1}\right\rangle \rightarrow\left|r_{1}^{2}\right\rangle \rightarrow\left|r_{2}^{2}\right\rangle \rightarrow\left|r_{3}^{2}\right\rangle \rightarrow\left|r_{4}^{1}\right\rangle$ & $(\omega, \omega, \omega) \rightarrow\left(\omega, \omega, \omega, \omega^{\prime}\right) \rightarrow\left(\omega, \omega, \omega^{\prime}\right) \rightarrow\left(\omega, \omega^{\prime}\right) \rightarrow\left(\omega^{\prime}\right)$ \\
\hline
\end{tabular}

Table 2. 


\begin{tabular}{|c|c|}
\hline Hyperpolarisability & Channel \\
\hline$\gamma_{x x x x}$ & $1,3,5,7$ \\
\hline$\gamma_{z(x z x)}$ & 4,6 \\
\hline$\gamma_{x z z x}$ & 2 \\
\hline$\gamma_{x x z z}$ & 8 \\
\hline$\gamma_{x(x z z)}$ & 2,8 \\
\hline
\end{tabular}

Table 3. 